\title{
Mindfulness in nursing students: The five facet mindfulness questionnaire in samples of nursing students in China, the Philippines, and South Africa
}

\author{
David Arthur, Doroteo Dizon, Karien Jooste, Zheng Li, Mathew Salvador and Xiuyu Yao
}

\begin{abstract}
Mindfulness as a clinical strategy for helping manage physical and psychological symptoms is gathering an impressive evidence base. It is also being embraced widely for personal and professional development. As a therapeutic intervention for nurses working across a spectrum of settings, it helps expand practice, and integrating mindfulness in nursing undergraduate and graduate curricula is imminent. In the present study, we outline the development and measurement of mindfulness as a personality trait and therapeutic intervention, and advocate the use of a questionnaire alternative to expensive and impractical laboratory measures. The aims of the present study were to examine and compare the use and reliability of the Five Facet Mindfulness Questionnaire (FFMQ) in samples of nursing students from South Africa, China, and the Philippines, and compare the findings in the nursing student samples to other student and non-student samples. Three samples of students of nursing from China $(\mathrm{n}=193)$, the Philippines $(n=243)$, and South Africa $(n=131)$ completed the 31-item FFMQ. The internal consistency was acceptable, and the correlations were significant among all facets and the total score. Although the total mean scores between the three samples were not significantly different, there were significant and interesting differences in the facet scores, and these are discussed in the light of likely cultural influences, and comparative data from other samples of students and clinical populations. The instrument shows promise for future use in educational research as a tool for curriculum change, for personal and professional development of nurses, and as a clinical tool for determining mindfulness changes over time.
\end{abstract}

\section{Introduction}

Mindfulness as a therapeutic intervention in mental health care is gathering momentum as a technique for improving well-being at least, and improving specific symptoms at best, and the evidence base is underpinning new developments in therapy. Its general, acceptance is influencing mental health nurses to make curriculum change and enhance professional growth through continuous professional development (Guillaumie et al. 2017; Hunter 2017).

If nursing programmes are to produce flexible graduates to meet the demands of modern health care, they should include mindfulness courses integrated into the curricula, 
as well as reliable and valid tools for determining the development of mindful characteristics in students. The argument for including mindfulness in nursing programmes is convincing, as it not only provides a therapeutic tool for individual, group, and family therapy, but also a tool for personal development. A nursing programme that produces mindful practitioners will enhance a health-care landscape that is expanding and increasingly taxing the abilities of its clinicians (Guillaumie et al. 2017).

In an attempt to explore more practical and useful measures of mindfulness for research and practice, the present study adds to the growing evidence base of mindfulness self-report measurement by comparing the results of three distinct groups of nursing students, one each in China, the Philippines and South Africa, on the Five Facet Mindfulness Questionnaire (FFMQ). As well as presenting comparative data, we also examined the psychometric characteristics in this sample of nursing students. The replication adds to the reliability and normative data base of the FFMQ.

\section{Background}

Mindfulness is a state in which a person is able to focus on the present, the surrounding environment and activities, and to concentrate on this without being distracted by thoughts about the past or the future (Guillaumie et al. 2017). Mindful people tend to be more focussed and relaxed, and are able to manage stress in a positive way.

Evidence-based strategies have found that by combining mindfulness with cognitive behaviour strategies, which help people recognize the effects of thoughts on emotion and vice versa, then promoting ways of changing thoughts to help improve emotions, such as anxiety and depression, actually help improve mental health (Kuyken et al. 2015). A recent rigorous study of mindfulness-based cognitive therapy (MBCT) compared to antidepressants in major depression over a 2-year period reached the understated conclusion that MBCT was not superior to medication (Kuyken et al. 2015). That is, it was as therapeutic as medication in this randomized, controlled trial of over 400 people. This was reinforced in a recent systematic review on the effects of mindfulness-based interventions on registered nurses and nursing students (Guillaumie et al. 2017), which revealed that mindfulness improved nurses' mental health, particularly anxiety and depression. The meta-analysis showed that mindfulness training seems to be effective, but the number of studies was small and lacked homogeneity, and more research needs to be conducted into workplace variables.

\section{Applications of mindfulness}

Systematic muscle relaxation and breathing exercises based on the stress-reduction model have been a component of relaxation techniques for decades, yet it is only relatively recently that mindfulness meditation has been adopted and identified as a technique with demonstrable brain changes to support its efficacy. Based on Zen and

\section{http://repository.uwc.ac.za}


Vipassana practices, it teaches how to focus attention on the present moment and how to acknowledge external and internal stimuli, yet to adopt non-judgmental acceptance of them (Kabat-Zinn 1982). Some authors refer to mindfulness as a trait or a disposition (Baer et al. 2006, 2011; Fernadez et al. 2010), where there are positive associations between mindfulness and self-esteem, quality of life, optimism, and satisfaction. Encouragingly, it is considered a trait which can be learnt.

Developments in neuroscience have shed light on the brain activities and the physiological basis of mindfulness, revealing the predominance of alpha brain waves, or the alpha brain state as the mechanism behind a meditative state (Sas \& Chopra 2015; Stinson \& Arthur 2013). Novel remote Electroencephalogram developments have enabled better understanding of how people can move from attentive into meditationlike states through mindfulness meditation and the technology for biofeedback training is available (Sas \& Chopra 2015; Stinson \& Arthur 2013). This technology remains expensive and impractical in many situations, thus the growing body of literature on self-report measures offers nurses in mental health, oncology, drug and alcohol, and beyond opportunities for self-development, expanding practice, and evaluative research.

The evidence is strong for the use of mindfulness-based stress reduction (MBSR), MBCT, and the 'third wave of cognitive behaviour therapy': mindfulness acceptance commitment therapy. MBSR and MBCT appeared in the literature in 1982 (Kabat-Zinn 1982) and 2003 (Baer 2003), respectively. Several recent studies using these techniques in various formats over differing time periods have been conducted with nurses and other health professionals receiving a mindfulness intervention (Guillaumie et al. 2017; Hunter 2017; Ratanasiripong et al. 2015; Song \& Lindquist 2015; Walker \& Mann 2016). Some success has been reported in improving outcome measures, such as anxiety, depression, and stress; however, few present more than a descriptive definition of mindfulness and what constitutes mindfulness as a construct is overlooked. Guillaumie et al. (2017) conducted a mixed-method systematic review of studies of mindfulness on nurses, in particular exploring what the main characteristics of these interventions were. Among the 32 studies, 13 psychological variables, such as depression and anxiety and stress; 12 different indicators of physical health; and four work-related variables, such as burnout and satisfaction, emerged. Again, little was reported on the measurement of mindfulness itself.

The following section examines the measurement of mindfulness through a review of the literature over the 10-year period ending in 2017, in which there has been a clear move from exploration of mindfulness as an alternative or adjunct, to mindfulness as a mainstream evidence-based technique for holistic intervention for a spectrum of symptoms. 


\section{FFMQ and measuring mindfulness}

Several publications on measuring mindfulness as a multifaceted concept have emerged, and in a landmark study, Baer et al. (2006) examined the construct validity of mindfulness in a non-meditating student sample by factor analysis of five mindfulness instruments, and proposed the items which offered a five-faceted model, which became the FFMQ. The five facets are 'observing', which relates to natural perception of the environment, such as sensing aromas and feeling wind and temperature changes. Items addressing translating feelings into words come under 'describing'. Items relating to judging oneself based on thoughts and feelings cluster under 'non-judging'; while 'non-reactivity' relates to managing distressing thoughts without overreacting; and 'act aware' items relate to focussing, rushing, and being attentive.

One of the earliest reviews of mindfulness focussed on the need to develop instruments to measure the effects of mindfulness meditation in intervention studies, in the context of MBSR and MBCT and mindfulness meditation. The present study of techniques for improving symptoms in cancer was authored by nurses (Matchim \& Armer 2007). They echoed the move, at the time, towards mindfulness as an 'alternative' therapy. The review covered the years from 1987 to 2006, revealing seven intervention studies which adopted MBSR as the intervention. The main outcomes were mood, stress and anxiety, health locus of control, helplessness and hopelessness, and sleep quality, all of which showed significant improvement. The studies were flawed by the use of 13 different instruments, three of which were used for the first time; only two were randomized, controlled trials, and most of the measures were not true measures of mindfulness as a construct, rather of psychological symptoms. Recommendations included further testing of measures for examining the relationship between symptoms and mindfulness in oncology samples.

Evidence of the FFMQ as a valid and reliable tool with normative data has steadily increased. Baer et al. (2006) examined the mean scores of meditators and nonmeditators, as well as correlations between mindfulness facets and psychological symptoms, concluding that mindfulness facets negatively correlated with mindfulness, and there were significant differences between meditators and non-meditators. Factor analysis supported the five-factor structure (Baer et al. 2006; Bohlmeijer et al. 2011) and suggests strong construct validity.

Additional psychometric analysis has emerged in the form of examination of whether negatively- or positively-stated items cause differential item functioning (Baer et al. 2011; Van Dam et al. 2009). Baer et al. (2011) report this as not problematic in matched demographic groups of meditators. Using the subscale scores, rather than a total scale scores, has been recommended (Bohlmeijer et al. 2011; Van Dam et al. 2012).

\section{http://repository.uwc.ac.za}




\section{Studies adopting the FFMQ}

The FFMQ has been translated into Dutch, Chinese, and Italian in six clinical studies, where it was tested in various student, patient, and community samples. Construct, convergent, and discriminant validity was examined in five studies, and reliability in all six of those studies (Table 1). Samples included patients with depressive symptomatology who participated in a self-help intervention using a waitlist control design (Bohlmeijer et al. 2011). All five facets of the FFMQ improved significantly in the treatment groups, and effect sizes were smallest for 'observing' and 'describing', and largest for 'non-judging' and 'non-reactivity'. The five-factor structure and correlations between psychological measures supported construct, convergent, and discriminant validity (Table 1).

In an attempt to develop a short form of the FFMQ with a Hong Kong Chinese sample, Hou et al. (2014) demonstrated similar evidence for the factor structure with good test-retest reliability and internal consistency, and proceeded to produce a 20-item short form of the scale. Unfortunately, it is unclear whether the translation was in simplified (Mainland Chinese preference) or traditional Chinese (commonly used in Hong Kong and Guangzou in Southern China).

Another study (Deng et al. 2011) in Mainland China translated the instrument into Chinese and explored its reliability and validity with a mixed sample of 246 undergraduates. The mean scores for facets were not reported, but a significant inverse correlation between the five facets (except for observing) and the Beck Depression Inventory and the State Trait Anxiety Inventory were established. Interestingly, the Cronbach alpha reliability estimates were lower than those of Baer et al. (2006), particularly 'non-react' (Cronbach a $=0.0448$ ), which could be explained by the fact that people in the East are more familiar with Buddhism and mindfulness concepts, and therefore, the concepts have different meanings depending where the instrument is administered. The authors recommend further cross-cultural differentiation of mindfulness (Deng et al. 2011), and conclude that the FFMQ is a valid instrument needing further testing, but with potential in different aspects of mindfulness training.

In an attempt to contribute to the cross-cultural adaptation of the FFMQ, it was translated into Italian (Giovannini et al. 2014) and subjected to reliability and validity testing with 355 undergraduate students and 281 non-student volunteers. All identified themselves as Italian and Caucasian. The findings revealed that the FFMQ and its multifacets suited the Italian context.

Mindfulness is emerging as having a positive therapeutic role in substance use disorders, yet the relationship between mindfulness and drug and alcohol use needs clarification, and instruments had yet to be tested when Fernadez et al. (2010) set out to examine the reliability and validity of the FFMQ in a sample of student problem drinkers. The five-facet model demonstrated in previous studies was supported, but

\section{http://repository.uwc.ac.za}


improved fit was found with removal of three 'describe' facet items, and there were negative correlations between mindfulness and alcohol use observed. The authors were cautious in drawing conclusions, and recommended further testing in experimental conditions. Another study aimed at examining the FFMQ and substance use among psychology students (Eisenlohr-Moul et al. 2012), and rather than relying on the five-facet structure alone, they examined interactions between the facets and found significant interactions. 'Observing' was associated with fewer periods of tobacco and alcohol use at higher levels of 'non-reactivity', and 'observing' was associated with more periods of heavy alcohol use at higher levels of 'non-reactivity'.

Taking the trait concept of mindfulness into the physiological domain, a study of 130 students was conducted. Resting blood pressure and the inflammatory marker interleukin6 (IL-6) and correlations with the facets of the FFMQ (Tomfohr et al. 2015) were recorded. Those who identified themselves as 'Asian' had lower mean FFMQ scores, IL-6 was associated with 'observing', and the regression analysis revealed that higher FFMQ scores were associated with lower mean blood pressure and IL-6 values, yet there were no significant interactions between the FFMQ scales and ethnicity, and mean blood pressure or IL-6. The authors re-analysed the relationships within the facets (Tomfohr et al. 2015) and hypothesized that higher levels of observation and/or awareness of the present moment might affect physiological outcomes only when paired with the "nonreactive or 'non-judgmental' facets. The study also reinforced the internal consistency of the FFMQ. Another study supported the psychometric features of the FFMQ (Curtiss \& Klemanski 2014). Associations with psychological constructs in a homogeneous clinical sample were found, and the results concurred with the other studies that found that the multifaceted structure is modified by psychopathology. In this clinical sample, excluding the 'observe' facet provided the best fit.

In their review of a decade of research on the FFMQ, Park et al. (2013) argued the importance of evaluating the measures of mindfulness as patient-reported outcomes. Overall, 2588 articles were reduced to 46 articles containing 79 separate studies evaluating 10 different mindfulness measures. Their results should be interpreted with caution, as they suggest that seven measures had moderate or strong positive results psychometrically, but they highlighted flaws with the instruments. 
TABLE 1: Validity and reliability of the FFMQ in different cultures and clinical studies

\begin{tabular}{|c|c|c|c|c|c|}
\hline Author & Sample & $\begin{array}{l}\text { Construct } \\
\text { validity }\end{array}$ & $\begin{array}{l}\text { Internal consistency } \\
\text { and correlations }\end{array}$ & $\begin{array}{l}\text { Convergent and } \\
\text { discriminant validity }\end{array}$ & Sensitivity to change \\
\hline $\begin{array}{l}\text { Bohlmeijer } \\
\text { et al. }(2011)\end{array}$ & $\begin{array}{l}\text { Depressed } \\
\text { Dutch } \\
\text { women }\end{array}$ & $\begin{array}{l}\text { CFA: } 5 \text { distinct } \\
\text { facets of } \\
\text { mindfulness }\end{array}$ & $\begin{array}{l}\alpha \text { coefficients } 0.73-0.91 \text {; } \\
\text { moderate correlation, } \\
\text { 'non-judge' not related } \\
\text { to observe; 'non-judge' } \\
\text { not related to describe }\end{array}$ & $\begin{array}{l}\text { Negative correlations with } \\
\text { anxiety and depression } \\
\text { (especially 'act aware' } \\
\text { and 'non-judge'); positive } \\
\text { correlations for acceptance, } \\
\text { especially 'non-judge'. } \\
\text { Positive mental health } \\
\text { relates most to observe, } \\
\text { describe, act aware, } \\
\text { non-judge and non-react }\end{array}$ & $\begin{array}{l}\text { All facets improved } \\
\text { significantly with } \\
\text { intervention with } \\
\text { moderate-to-large } \\
\text { effect sizes (except } \\
\text { for 'describe') }\end{array}$ \\
\hline $\begin{array}{l}\text { Deng et al. } \\
\text { (2011) }\end{array}$ & $\begin{array}{l}\text { University } \\
\text { students, } \\
\text { Mainland } \\
\text { China }\end{array}$ & $\begin{array}{l}\text { CFA: goodness- } \\
\text { of fit-indices } \\
\text { supported } \\
\text { 5-factor model }\end{array}$ & $\begin{array}{l}\alpha \text { coefficients } 0.74-0.84 \\
\text { ('non-react' }=0.45) \\
\text { Test-retest coefficient: } \\
0.44-0.74(P<0.01)\end{array}$ & Not reported & Not reported \\
\hline $\begin{array}{l}\text { Giovannini } \\
\text { et al. }(2014)\end{array}$ & $\begin{array}{l}\text { Italian } \\
\text { students } \\
\text { and adults }\end{array}$ & $\begin{array}{l}\text { CFA: goodness- } \\
\text { of fit-indices } \\
\text { supported five } \\
\text { factor model. } \\
\text { All factor } \\
\text { loadings }>0.30\end{array}$ & $\begin{array}{l}\alpha \text { coefficients }>0.74 \\
\text { Facets correlated } \\
\text { positively with total } \\
\text { FFMQ score; 'observe' } \\
\text { negatively correlated with } \\
\text { 'non-judge' and 'act aware' } \\
\text { Test-retest: scale ICC: } \\
0.71 \text {, subscales } 0.63-0.81\end{array}$ & $\begin{array}{l}\text { 'Act aware' negative } \\
\text { correlated with } \\
\text { dissociation scale; } \\
\text { 'non-judge' correlated } \\
\text { with a tendency to } \\
\text { dissociation and } \\
\text { thought suppression; } \\
\text { 'describe' negatively } \\
\text { associated with } \\
\text { alexithymia and } \\
\text { positive emotional } \\
\text { awareness; 'non-react' } \\
\text { correlated with } \\
\text { emotional stability; } \\
\text { 'observe' positively } \\
\text { correlated with } \\
\text { mental openness } \\
\text { and emotional } \\
\text { awareness, } \\
\text { dissociation, } \\
\text { and thought } \\
\text { suppression }\end{array}$ & Not reported \\
\hline $\begin{array}{l}\text { Fernadez } \\
\text { et al. }(2010)\end{array}$ & $\begin{array}{l}\text { US } \\
\text { university } \\
\text { students }\end{array}$ & $\begin{array}{l}\chi^{2} \text { and } \\
\text { goodness-of-fit } \\
\text { test. } \\
\text { Five-factor } \\
\text { model supported, } \\
\text { three 'describe' } \\
\text { items removed }\end{array}$ & $\alpha$ coefficients $0.70-0.90$ & $\begin{array}{l}\text { Negative associations } \\
\text { between alcohol use } \\
\text { and 'act aware' } \\
\text { and 'describe' }\end{array}$ & Not reported \\
\hline $\begin{array}{l}\text { Eisenlohr-Moul } \\
\text { et al. }(2012)\end{array}$ & $\begin{array}{l}\text { US } \\
\text { Psychology } \\
\text { students }\end{array}$ & Not reported & $\begin{array}{l}\text { Only 'observe', } \\
\text { 'non-judge', and } \\
\text { 'non-react' subscales: } \\
\alpha \text { coefficients } 0.70-0.88\end{array}$ & $\begin{array}{l}\text { 'Observe' associated with } \\
\text { fewer periods of tobacco } \\
\text { and alcohol use only at } \\
\text { higher levels of 'non-react'; } \\
\text { 'observe' associated with } \\
\text { heavy alcohol use at lower } \\
\text { levels of 'non-react', but less } \\
\text { heavy alcohol use at higher } \\
\text { levels of 'non-react' }\end{array}$ & Not reported \\
\hline
\end{tabular}


TABLE 1: (Continued)

\begin{tabular}{|c|c|c|c|c|c|}
\hline Author & Sample & $\begin{array}{l}\text { Construct } \\
\text { validity }\end{array}$ & $\begin{array}{l}\text { Internal consistency } \\
\text { and correlations }\end{array}$ & $\begin{array}{l}\text { Convergent and } \\
\text { discriminant validity }\end{array}$ & Sensitivity to change \\
\hline $\begin{array}{l}\text { Tomfohr et al. } \\
\text { (2015) }\end{array}$ & US students & Not reported & $\begin{array}{l}\text { Except for 'observe', } \\
\text { facets and FFMQ } \\
\text { mean significant, } \\
\text { positive association } \\
\text { with each other }\end{array}$ & $\begin{array}{l}\text { Negative correlation with } \\
\text { depression and perceived } \\
\text { stress interleukin- } 6 \text { negatively } \\
\text { associated with 'observe' (i.e. } \\
\text { higher FFMQ associated with } \\
\text { lower blood pressure) }\end{array}$ & Not reported \\
\hline
\end{tabular}

CFA, confirmatory factor analysis; FFMQ, Five Facet Mindfulness Questionnaire; ICC, Intraclass Correlation Coefficient.

In view of the strength of the FFMQ and the flexibility offered by the five facets, we decided to proceed with using it in the present study and add some context for future nursing research and practice.

The FFMQ shows promise as a measure of the multidimensional construct mindfulness and as a tool for measuring the effectiveness of mindfulness interventions in clinical and non-clinical populations. There is good reliability evidence, and face, content, and construct validity have been well examined in psychometric studies; however, more work is needed on concurrent and predictive validity. Because mindfulness and hypnosis in Western thought have been considered adjunctive or complementary therapies, at best, measures of mindfulness beyond the subjective are in their infancy.

Mindfulness is being introduced into nursing practice, and should be a component of curricula in multi-cultural countries where immigration and international students from diverse countries practice and study. Students from China, the Philippines, and Africa study and practice nursing in many countries where consumers come from similar cultural backgrounds. Therefore, having a tool that measures the impact of mindfulness programmes on nursing students, which can also claim cross-cultural sensitivity, as well as reliability, will be valuable.

The objectives of the present study were to:

(i) examine and compare the use and reliability of the FFMQ in samples of nursing students from South Africa, China, and the Philippines; and (ii) compare the findings to other student and non-student samples.

\section{Methods}

A quantitative survey design was adopted with a convenience sample.

\section{Participants}

The convenience sample consisted of 193 Bachelor of Science (Nursing) (BSN) students enrolled in one of Beijing's top-ranked universities; 243 BSN students from a highlyranked Filipino university, and 131 BSN students from a similarly-ranked university in South Africa. This non-probability convenience sampling method was chosen because of

\section{http://repository.uwc.ac.za}


convenient accessibility and proximity to the research team who were in different countries, but all interested in exploring this topic. No demographic data were collected; rather, the assumption was made that these were healthy adults, the majority $<25$ years of age, in their final year, aiming at a career in nursing.

Following ethics approval from the host university, volunteer participants completed the questionnaires in class time, and placed the completed questionnaires in collection boxes. Those that were not completed were removed before the final analysis.

\section{Instrument}

The FFMQ consists of 39 items on a five-point Likert scale, with high scores reflecting a higher degree of mindfulness, which is increasingly recognized as a trait-like characteristic. A Mandarin Chinese version of the FFMQ was developed by the Beijing authors during several years of unreported pilot work, by translating the original (Baer et al. 2006) into Chinese for the Beijing sample. Independent forward-translation by the bilingual authors preceded back-translation to ensure accuracy. The Beijing team are well versed in this procedure, and utilized experts in English and translation to ensure accuracy and validity of translation. The English version (Baer et al. 2006) was used for both the Filipino and South African sample whose medium of study was English.

The five facets (or subscales) of the mindfulness construct are: 'describe' (e.g. 'I am good at finding words to describe my feelings') 'act aware' (e.g. 'I find it difficult to stay focussed on what's happening in the present'), 'non-judge' (e.g. 'I criticize myself for having irrational or inappropriate emotions', 'non-react' (e.g. 'I perceive my feelings and emotions without having to react to them'), and 'observe' (e.g. 'when I am walking, I deliberately notice the sensations of my body moving' (Baer et al. 2006). As noted, the instrument has demonstrated good internal consistency for the total scale and subscales, test-retest reliability, convergent and divergent validity, construct validity, and sensitivity to different samples, such as meditators and nonmediators and clinical samples (Baer et al. 2006, 2008), and more recently in different languages and cultures (Deng et al. 2011; Giovannini et al. 2014; Hou et al. 2014) (Table 2).

\section{Data analysis}

Descriptive statistics were used to compare the distributions of scores from the three samples with those from other studies, and inferential procedures examined internal consistency, correlations of scales, and subscales within samples. Negative item scores were reversed to reflect high scores being higher in mindfulness.

The mean scores for the FFMQ and the sub-scale were compared to those from several other studies in order to present a norm reference for comparison of samples from different cultures, meditating background, and now the nursing profession for

\section{http://repository.uwc.ac.za}


future comparative purposes. The means scores for the 39-item FFMQ and five facets in each of the three samples were compared using analysis of variance.

\section{Results}

The three datasets were analysed separately to examine differences between mean scores, correlation coefficients, and Cronbach alpha coefficient for the total FFMQ and for each of the five facets (Table 1). Satisfactory overall internal consistency was established for the 39 items in all three samples. At the facet level, 'non-react' was less than the conventional Cronbach alpha of 0.70 level all three samples. In the Chinese and South African samples, the other facets had scores ranging from 0.70 to 0.90 , supporting the internal consistency of the FFMQ. However, the reliability in the Filipino sample was less convincing, as although all facets significantly correlated with the total FFMQ score, the rest ranged from 0.24 to 0.60 . However the 'Cronbach alpha if item removed' statistic revealed that removing items 16 ('non-react' to Cronbach a $=0.68$ ), 17 ('observe' to Cronbach $\mathrm{a}=0.69$ ), 33 ('act aware' to Cronbach $\mathrm{a}=0.66$ ), and 20 ('non-judge' to Cronbach $\mathrm{a}=0.49$ ) would improve reliability to more tolerable standards at the facet levels. 'Describe' remained problematic in terms of internal consistency.

There were significant positive correlations $(\mathrm{P}<0.001)$ between all facets and the total FFMQ in all three samples (Table 3). The strength of correlations between the overall FFMQ scores and the each of the facets was calculated for each of the three samples. The correlations in the Filipino sample were statistically significant (Table 3), and ranged from 0.58 to 0.73 , indicating a strong relationship between each of the facets and the instrument as a whole. The South African sample correlations were similar, ranging from 0.50 to 0.79 (i.e. moderate to strong), again suggesting satisfactory internal structural relationships. However, while still significant, the Beijing sample correlations ranged from weak ( 0.21 for 'non-judge') to moderate for the remaining facets. This suggests some problematic areas in the instrument, except for 'act aware', the scores for items and facets in the Beijing sample were weaker than the other two samples, suggesting a cultural influence or different understanding of meanings of the mindfulness concepts.

TABLE 2: Analysis of variance scores for nursing student samples

\begin{tabular}{|c|c|c|c|c|c|c|}
\hline Facets & No. Items & $\begin{array}{l}\text { The } \\
\text { Philippines (A) }\end{array}$ & $\begin{array}{c}\text { South } \\
\text { Africa (B) }\end{array}$ & $\begin{array}{l}\text { China } \\
\text { (C) }\end{array}$ & F-score & $\begin{array}{c}\text { Tukey's } \\
\text { post-hoc test }\end{array}$ \\
\hline FFMQ & 39 & 120.98 & 123.52 & 123.68 & 2.98 & ns \\
\hline All non-react & 7 & 22.61 & 21.2 & 21.8 & 6.2 & $\mathrm{AB}^{*}$ \\
\hline All observe & 8 & 25.96 & 26.76 & 25.58 & 2.36 & ns \\
\hline All act aware & 8 & 21.94 & 23.29 & 23.45 & 8.71 & $\mathrm{AB}^{*}, \mathrm{AC}^{*}$ \\
\hline All describe & 8 & 24.08 & 25.88 & 27.01 & 27.18 & $\begin{array}{l}\mathrm{AB}^{*}, \mathrm{AC}^{*}, \mathrm{BA}^{*} \\
\mathrm{BC}^{*}, \mathrm{CA}^{*}, \mathrm{CB}^{\circ}\end{array}$ \\
\hline All non-judge & 8 & 23.16 & 23.55 & 22.67 & 1.73 & ns \\
\hline
\end{tabular}

A B C: different letters indicate significant difference. ${ }^{*}$ Indicates significance at the 0.05 level.

FFMQ, Five Facet Mindfulness Questionnaire; ns, not significant. 
TABLE 3: Correlation matrix and Cronbach alpha coefficients of facets for nursing student samples

\begin{tabular}{|c|c|c|c|c|c|c|c|c|c|}
\hline & Item no. & Cronbach $\alpha$ & Mean & SD & Observe & Describe & Act aware & Non-judge & Non-react \\
\hline \multicolumn{10}{|c|}{ Filipino sample $(n=243)$} \\
\hline Observe & 8 & 0.56 & 25.96 & 4.02 & - & - & - & - & - \\
\hline Describe & 8 & 0.24 & 24.08 & 2.46 & $0.34^{\circ \circ}$ & - & - & - & - \\
\hline Act aware & 8 & 0.60 & 21.94 & 4.11 & $0.38^{\circ} \circ$ & $0.41^{\circ 0}$ & - & - & - \\
\hline Non-judge & 8 & 0.31 & 23.61 & 4.02 & $0.20^{\circ \circ}$ & $0.19^{\circ \circ}$ & $0.44^{\circ \circ}$ & - & - \\
\hline Non-react & 7 & 0.58 & 22.61 & 4.11 & $0.29^{\circ} \circ$ & $0.23^{\circ} \circ$ & 0.70 & 0.05 & - \\
\hline FFMQ & 39 & 0.74 & 120.98 & 9.36 & $0.70^{\circ \circ}$ & $0.58^{\circ \circ}$ & $0.73^{\circ \circ}$ & $0.58^{\circ \circ}$ & $0.70^{\circ \circ}$ \\
\hline \multicolumn{10}{|c|}{ Beijing sample $(n=193)$} \\
\hline Observe & & 0.79 & 25.58 & 4.86 & - & - & - & - & - \\
\hline Describe & & 0.84 & 27.01 & 4.41 & 0.14 & - & - & - & - \\
\hline Act aware & & 0.84 & 23.45 & 4.05 & $-0.15^{\circ}$ & $0.33^{\circ 0}$ & - & - & - \\
\hline Non-judge & & 0.90 & 22.67 & 3.57 & $-0.44^{\circ \circ}$ & 0.22 & $0.35^{\circ 0}$ & - & - \\
\hline Non-react & & 0.58 & 21.80 & 3.02 & $0.15^{\circ}$ & 0.08 & 0.3 & $-0.29^{\circ \circ}$ & - \\
\hline FFMQ & & 0.73 & 123.69 & 9.54 & $0.39^{\circ \circ}$ & $0.36^{\circ \circ}$ & $0.64^{\circ \circ}$ & $0.21^{\circ 0}$ & $0.36^{\circ \circ}$ \\
\hline \multicolumn{10}{|c|}{ South African sample $(n=131)$} \\
\hline Observe & & 0.70 & 26.76 & 5.20 & - & - & - & - & - \\
\hline Describe & & 0.70 & 25.88 & 5.27 & $0.50^{\circ \circ}$ & - & - & - & - \\
\hline Act aware & & 0.77 & 23.29 & 5.80 & $0.27^{\circ \circ}$ & $0.61^{\circ \circ}$ & - & - & - \\
\hline Non-judge & & 0.80 & 23.55 & 5.80 & -0.11 & 0.15 & $0.40^{\circ \circ}$ & - & - \\
\hline Non-react & & 0.61 & 21.20 & 4.34 & $0.40^{\circ \circ}$ & $0.24^{\circ \circ}$ & $0.21^{\circ}$ & -0.05 & - \\
\hline FFMQ & & 0.75 & 123.52 & 16.78 & $0.61^{\circ 0}$ & $.074^{\circ \circ}$ & $0.79^{\circ \circ}$ & $0.51^{\circ \circ}$ & $0.50^{\circ \circ}$ \\
\hline
\end{tabular}

${ }^{\circ} \mathrm{P}<0.01,{ }^{\circ} \mathrm{P}<0.001$.

FFMQ, Five Facet Mindfulness Questionnaire; SD, standard deviation.

In the South African sample, there were significant correlations between all facets, except 'non-judge', which negatively correlated with 'non-react', and 'observe' and was weak with 'describe'. In the Filipino sample, all were significantly correlated across the matrix' except for 'non-react', which showed weak correlations with 'act aware' and 'non-judge'. In the Chinese sample, there were weak correlations between 'non-react', 'observe', and 'nonjudge'; 'act aware' and 'non-judge'; and 'observe' and 'describe' (Table 4).

The data from all three samples were normally distributed. The mean scores (standard deviations) at the total and facet level showed some interesting trends across the 10 samples (Table 4). Clearly, the meditators and highly educated (Baer et al. 2006) showed consistently higher mean scores. Our Chinese sample of nursing students showed higher mean scores in all but the 'non-judge' facet when compared to the psychology students from Hong Kong (Hou et al. 2014) (Table 4). Apart from the 'non-judge' scores in the Italian (Giovannini et al. 2014) and US samples (Baer et al. 2006), which were noticeable higher, and peaking with meditators. All other scores showed similarities and differences suggestive of the sensitivity of the instrument.

Comparing the mean scores for the total FFMQ revealed no significant differences between the Chinese, Filipino, and South African samples. However, at the facet level, the difference between the mean scores for all three sites on 'observe' was not significant; for 'non-react', there was only a difference between the South African and Filipino samples; for 'act aware', there was no difference between the Chinese and South African samples; for 'describe', there was a significant difference in all mean scores; and for 'non-judge', no differences were noted between any of the samples (Table 4). That is, the similarity between means suggested that there was homogeneity in terms of the 39-

\section{http://repository.uwc.ac.za}


item FFMQ. The Chinese were different to the others in terms of 'non-react', the Filipinos were different in their interpretation of 'act aware', there was a difference between all three groups in their interpretation of 'describe', and there was a tendency towards agreement on 'non-judge'.

\section{Discussion}

The results of the present study provide useful evidence that the FFMQ is reliable and valid in different cultures (Deng et al. 2011; Giovannini et al. 2014; Hou et al. 2014), with Italian students reported as more mindful than Chinese (Deng et al. 2011 \& Hou et al. 2014), as well the samples of students in the present study. The instrument proved internally consistent in the South African and Chinese samples, and acceptable in the Filipino sample if four items were removed. This suggests that interpretation of some items is problematic in the Filipino sample, particularly in the 'describe' facet, which is essentially about expressing feelings in words with ease. This could be related to cultural characteristics about expressing one's feelings to others. If the Asian stereotype is correct, that is emotional reserve, then this might help explain the difference between South Africans and Filipinos, but not Chinese, as they are often referred to as reserved people when it comes to expressing emotions. The Chinese students in the present study scored significantly higher in the 'describe' facet, which should be a reflection of a cultural characteristic, that is, of being at ease with expressing one's emotions, but clearly this needs further exploration and was not clarified in the previous studies of Hou et al. (2014) or Deng et al. (2011). The mean scores differed between each group, suggesting that the ability to express emotion is a facet of the FFMQ that differentiates and is sensitive to cultural differences. 


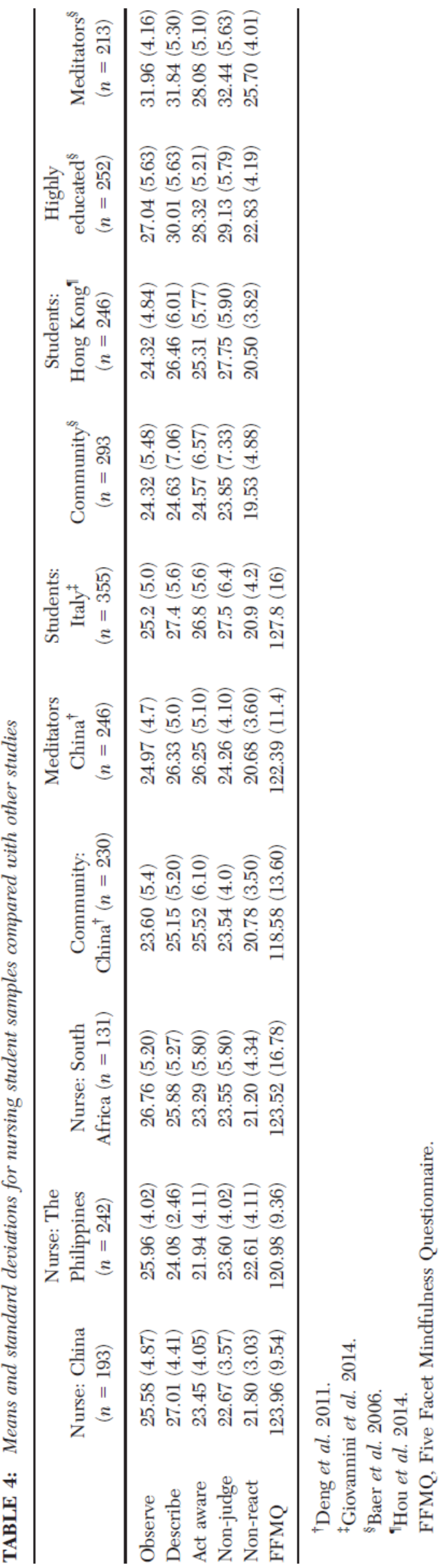

http://repository.uwc.ac.za 
The items in the 'non-react' facet relate to the ability to accept thoughts and images without being distressed or upset by them, and being able to recognize such thoughts and let them go. This was less reliable that the other facets across all three groups. This concept is a cornerstone of mindfulness (Baer et al. 2011), and might be less reliable among less mindful people; therefore, inconsistency of response would be expected. The Filipino group was more mindful of this facet than both other groups, and was different at the significance level with the South African group.

The items relating to 'observe' highlight the very important mindful characteristic of being present, alert, and enjoying sensations and perception of activities and nature (Guillaumie et al. 2017; Hunter 2017). There was a poor correlation between 'observe' and 'non-judge' in the South African and Chinese samples, and overall there was a tendency for the characteristic to be similar among the groups. Similar to that reported by Baer et al. (2006) and Bohlmeijer et al. (2011), 'non-judging' was not related to observing, and in Bohlmeijer et al.'s (2011), study 'non-judge' was also unrelated to 'describing'. Interestingly, Giovannini et al. (2014) found that the facets in the Italian sample were not strongly correlated among themselves, yet positively and significantly correlated with the total FFMQ score.

The items comprising the 'act aware' facet (Baer et al. 2011) relate to the ability to stay focussed on the present, a sense of awareness, or attention to activities without becoming distracted. With reasonable reliability estimates, in both the Chinese and Filipino groups there was a weak correlation between 'act aware' and 'non-judge', which is the mindful characteristic of being critical of thoughts or emotions and judging one's self harshly or negatively for having such thoughts. The homogeneity of the facets in terms of mean score suggests similarity in the three groups' mindfulness in this facet.

The internal consistency was acceptable, the correlations significant among all facets and the total FFMQ, and there was sensitivity in the facets, consistent with other studies (Baer et al. 2006, 2008). Of note, there were some negative correlations between the 'act aware' and 'observe', and 'non-judge' and 'observe', and 'non-judge' and 'non-react' in the Beijing sample, which were consistent with those reported in the Italian sample. There were non-significant correlations for 'observe' with 'describe', 'act aware', and 'non-react', and for 'non-react' with 'observe', 'describe', and 'act aware'. The Filipino sample correlations were all positive and significant.

The mean scores for the three samples showed the Chinese sample to have the highest FFMQ score (not significant), and were more mindful in terms of 'act aware' and 'describe'; the Filipino group had the highest 'non-react'; and the South African group had the highest 'observe' score. When comparing the data to those of Baer et al. (2006) and Hou et al. (2014), there was a tendency for scores to improve in all facets with increases in education 
level and meditation. In the Hou et al. (2014) sample, the scores in meditators were noticeable lower than those in the Baer et al. (2006) sample.

Baer et al. (2006) reported mean difference for the five facets for samples of students, community, highly educated, and meditators, and found significant $(\mathrm{P}<0.0001)$ differences between meditators and all groups on all facets. Between meditators and highly educated, there were significant differences $(\mathrm{P}<0.0001)$ on the 'observe', 'non-judge', and 'non-react' and 'describe' $(\mathrm{P}<\mathrm{0.001})$ facets, and no significant differences for 'act aware'.

\section{Conclusion}

Mindfulness is gathering momentum in practice and popularity as a research topic. Previously considered an adjunct or complementary to mainstream practice, the evidence base is now sufficiently broad and deep to be convincing that mindfulness meditation and mindfulness based interventions have applications across a spectrum of psychological and physical disorders (Guillaumie et al. 2017; Hunter 2017). Guillaumie et al. (2017) emphasize the need to explore more psychological variables and work behavioural changes, as well as longer-term impacts, maintenance, and sustainability of mindfulness. They also note methodological limitations, such as content of sessions and mindfulness therapists' backgrounds, selection bias, and the small number of studies.

Measuring mindfulness has become popular, but unfortunately, as is often the case in health care, there is an abundance of studies reporting on the development of new instruments and insufficient reporting replication and testing of pen and paper instruments, which have concurrent validity. As the FFMQ was developed in a rigorous study (Baer et al. 2006), it has been adopted in several studies, and its evidence as a valid and reliable measure is growing.

The present study was confined to comparing the FFMQ descriptive and reliability results in nursing samples in different countries and cultures. Further studies to establish concurrent validity are needed, and as remote electro-encephalography technology advances, this will become an easier mechanism for exploring the relationship between the FFMQ and other previously-established measures. Similarly, more longitudinal and correlational studies utilizing selected, reliable, and valid outcome measures are necessary to help establish the predictive validity of the FFMQ.

A limitation of the present study was the lack of demographic data to enable stratification of the sample to examine correlations between factors, such as age and sex, and importantly, between different years of the BSN programme. It is hypothesized that mindfulness scores improve as a student progresses in a nursing programme, and that learning mindfulness practice as part of a nursing programme enhances well-being; however, this need to be tested, and is part of this research team's agenda moving forward. 
A further limitation was selecting a non-random, convenience sample and not justifying the sample size based on previous studies, rather than by an a priori analysis. This is reported as a problem in such research (Anthoine et al. 2014), and in factor analyses, is usually guided by a subject-to-item ratio, which also varies, but is conventionally acceptable at 3:5.

\section{Relevance for clinical practice}

Enhancing nursing curricula with mindfulness training for personal and professional growth, and introducing mindfulness as a therapeutic option for nurses across a spectrum of disorders and symptoms, are considered beneficial innovations. There is no doubt that healthcare needs worldwide will demand a more mindful professional, and future research and curriculum development should benefit from such innovations. Guillaumie et al. (2017) in their systematic review, and Hunter's (2017) commentary on this systematic review, conclude by claiming that mindfulness promises characteristics, such as awareness, serenity, empathy, and inner calmness. Hunter (2017) further suggests that making time and saving space are dominant themes that enables nurses to improve their listening, increase attentiveness, and be less judgmental, that is, improved caring. 


\section{References}

Anthoine, E., Moret, L., Regnault, A., S'ebille, V. \& Hardouin, J. (2014). Sample size used to validate a scale: A review of publications on newly-developed patient reported outcomes measures. Health Quality Life Outcomes, 12, 2.

Baer, R. A. (2003). Mindfulness training as a clinical intervention: A conceptual and empirical review. Clinical Psychology Science and Practice, 10, 125-143.

Baer, R. A., Smith, G. T., Hopkins, J. et al. (2006). Using self-report assessment methods to explore facets of mindfulness. Assessment, 13, 27-45.

Baer, R. A., Smith, G. T., Lykins, E. et al. (2008). Construct validity of the five-facet mindfulness questionnaire in meditating and non-meditating samples. Assessment, $15,329-342$.

Baer, R. A., Samual, D. B. \& Lykins, E. L. B. (2011). Differential item functioning on the five-facet mindfulness questionnaire is minimal in demographically matched meditators and non meditators. Assessment, 18, 3-10.

Bohlmeijer, E., ten Klooster, P. M., Fledderus, M., Veehof, M. \& Baer, R. (2011). Psychometric properties of the five-facet mindfulness questionnaire in depressed adults and development of a short form. Assessment, 18, 308-320.

Curtiss, J. \& Klemanski, D. (2014). Factor analysis of the five-facet mindfulness questionnaire in a heterogeneous clinical sample. Journal of Behavioural Assessment, 36, 683-694.

Deng, Y. Q., Liu, H. H., Rodrigeuz, M. A. \& Xia, C. Y. (2011). The five facet mindfulness questionnaire: Psychometric properties of the Chinese version. Mindfulness, 2, 123128.

Eisenlohr-Moul, T. A., Walsh, E. C., Charnigo, R. J., Lynam, D. R. \& Baer, R. A. (2012). The 'what' and 'how' of dispositional mindfulness: Using interactions among subscales of the five-facet mindfulness questionnaire to understand its relation to substance abuse. Assessment, 19, 276-286.

Fernadez, A., Wood, M., Stein, L. \& Rossi, J. (2010). Measuring mindfulness and examining its relationship with alcohol use and negative consequences. Psychology of Addictive Behaviours, 24, 608-616.

Giovannini, C., Giromini, L., Bonalume, L., Tagini, A., Lang, M. \& Amadei, G. (2014). The Italian five-facet mindfulness questionnaire: A contribution to its validity and reliability. Journal of Psychopathology and Behavioural Assessment, 36, 415-423.

Guillaumie, L., Boiral, O. \& Champagne, J. (2017). A mixed-methods systematic review of the effects of mindfulness on nurses. Journal of Advanced Nursing, 73, 1017-1034. https://doi.org/10.1111/jan.13176.

Hou, J., Wong, S. Y.-S., Lo, H. H.-Y., Mak, W. W.-Z. \& Ma, H. S.-W. (2014). Validation of a Chinese version of the five-facet mindfulness questionnaire in Hong Kong and development of a short form. Assessment, 21, 363-371.

Hunter, L. (2017). Mindfulness training can reduce depression and anxiety among nurses. Evidence Based Nursing, 20, 57. 
Kabat-Zinn, J. (1982). An outpatient program in behavioural medicine for chronic pain patients based on the practice of mindfulness meditation: Theoretical consideration and preliminary results. General Hospital Psychiatry, 4, 33-42.

Kuyken, W., Hayes, R., Barrett, B. et al. (2015). Effectiveness and costeffectiveness of mindfulness-based cognitive therapy compared with maintenance antidepressant treatment in the prevention of depressive relapse or recurrence (PREVENT): A randomised controlled trial. The Lancet, 386, 63-73.

Matchim, Y. \& Armer, J. (2007). Measuring the psychological impact of mindfulness meditation on health among patiens with cancer: A literature review. Oncology Nursing Forum, 34, 1059-1066.

Park, T., Reilly-Sponmg, M. \& Gross, C. (2013). A systematic review of measurement properties of mindfulness instruments. Quality of Life Research, 22, 2639-2659.

Ratanasiripong, N., Park, J. \& Duangrat, K. (2015). Stress and anxiety management in nursing students: Biofeedback and mindfulness meditation. Journal of Nursing Education, 54, 520-524.

Sas, C. \& Chopra, R. (2015). MeditAid: A wearable adaptive neurofeedback-based system for training mindfulness state. Personal and Ubiquitous Computing, 19, 1169-1182. Song, Y. \& Lindquist, R. (2015). Effects of mindfulness-based stress reduction on depression, anxiety, stress and mindfulness in Korean nursing students. Nurse Education Today, 35, 86-90.

Stinson, B. \& Arthur, D. (2013). A novel EEG for alpha brain state training, neurobiofeedback and behavior change. Complementary Therapies in Clinical Practice, 19, 114-118.

Tomfohr, H. M., Pung, M. A., Mills, P. J. \& Edwards, K. (2015). Trait mindfulness is associated with blood pressure and interleukin-6: Exploring interactions among subscales on the Five Facet Mindfulness Questionnaire to better understand relationships between mindfulness and health. Journal of Behavioural Medicine, 38, 28-38.

Van Dam, N. T., Earlywine, M. \& Danoff-Burg, S. (2009). Differential item functioning across meditators and non meditators on the Five Facet Mindfulness Questionnaire. Personality and Individual Differences, 47, 516-521.

Van Dam, N., Hobkirk, A. L., Danoff-Burg, S. \& Earlywine, M. (2012). Mind your words: Positive and negative items create method effects on the five facet mindfulness questionnaire. Assessment, 19, 198-204.

Walker, M. \& Mann, R. (2016). Exploration of mindfulness in relation to compassion, empathy and reflection within nursing education. Nurse Education Today, 40, 188-190. 